\title{
Molecular simulations of heterogeneous ice nucleation. II. Peeling back the layers
}

Stephen J. Cox, Shawn M. Kathmann, Ben Slater, and Angelos Michaelides'

Citation: The Journal of Chemical Physics 142, 184705 (2015); doi: 10.1063/1.4919715

View online: http://dx.doi.org/10.1063/1.4919715

View Table of Contents: http://aip.scitation.org/toc/jcp/142/18

Published by the American Institute of Physics

\section{Articles you may be interested in}

Molecular simulations of heterogeneous ice nucleation. I. Controlling ice nucleation through surface hydrophilicity

The Journal of Chemical Physics 142, 184704184704 (2015); 10.1063/1.4919714

Pre-ordering of interfacial water in the pathway of heterogeneous ice nucleation does not lead to a two-step crystallization mechanism

The Journal of Chemical Physics 145, 211910211910 (2016); 10.1063/1.4961652

Ice formation on kaolinite: Insights from molecular dynamics simulations

The Journal of Chemical Physics 145, 211927211927 (2016); 10.1063/1.4968796

The surface charge distribution affects the ice nucleating efficiency of silver iodide

The Journal of Chemical Physics 145, 211924211924 (2016); 10.1063/1.4966018

Effects of surface interactions on heterogeneous ice nucleation for a monatomic water model

The Journal of Chemical Physics 141, 084501084501 (2014); 10.1063/1.4892804

Note: Heterogeneous ice nucleation on silver-iodide-like surfaces

The Journal of Chemical Physics 141, 216101216101 (2014); 10.1063/1.4902382

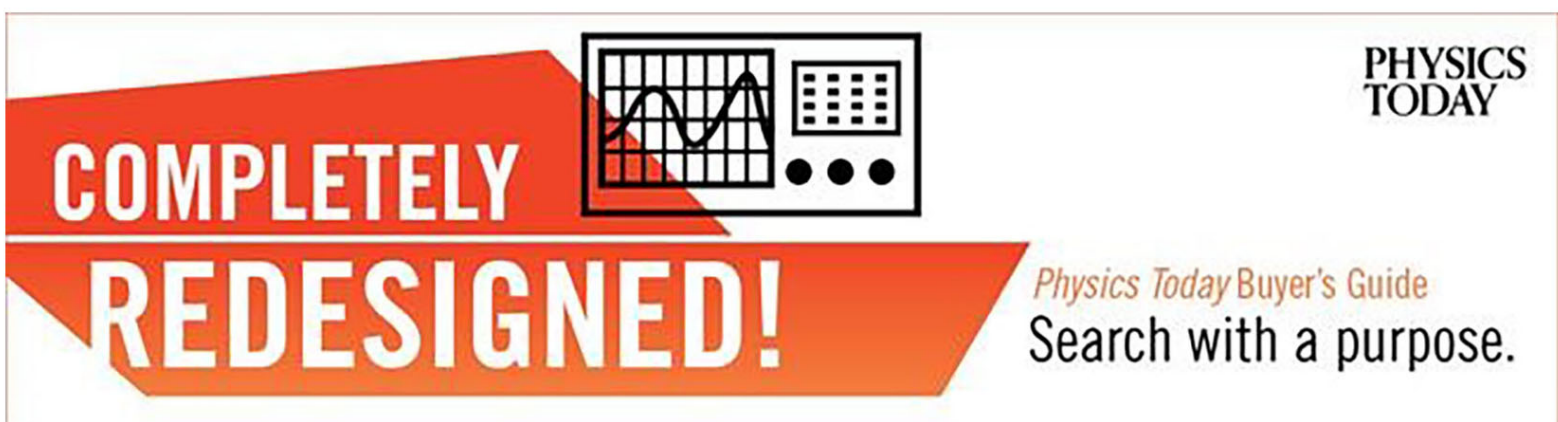




\title{
Molecular simulations of heterogeneous ice nucleation. II. Peeling back the layers
}

\author{
Stephen J. Cox, ${ }^{1,2}$ Shawn M. Kathmann, ${ }^{3}$ Ben Slater, ${ }^{1}$ and Angelos Michaelides ${ }^{1,2, a)}$ \\ ${ }^{1}$ Thomas Young Centre and Department of Chemistry, University College London, 20 Gordon Street, \\ London WC1H OAJ, United Kingdom \\ ${ }^{2}$ London Centre for Nanotechnology, 17-19 Gordon Street, London WC1H OAH, United Kingdom \\ ${ }^{3}$ Physical Sciences Division, Pacific Northwest National Laboratory, Richland, Washington 99352, USA
}

(Received 8 January 2015; accepted 22 April 2015; published online 12 May 2015)

Coarse grained molecular dynamics simulations are presented in which the sensitivity of the ice nucleation rate to the hydrophilicity of a graphene nanoflake is investigated. We find that an optimal interaction strength for promoting ice nucleation exists, which coincides with that found previously for a face centered cubic (111) surface. We further investigate the role that the layering of interfacial water plays in heterogeneous ice nucleation and demonstrate that the extent of layering is not a good indicator of ice nucleating ability for all surfaces. Our results suggest that to be an efficient ice nucleating agent, a surface should not bind water too strongly if it is able to accommodate high coverages of water. (C) 2015 Author(s). All article content, except where otherwise noted, is licensed under a Creative Commons Attribution 3.0 Unported License. [http://dx.doi.org/10.1063/1.4919715]

\section{INTRODUCTION}

As liquid water is cooled below its melting point, it crystallizes to solid ice. This familiar yet important process is not fully understood, especially at the molecular level. It is known that pure water can exist in the liquid state far below $0{ }^{\circ} \mathrm{C}$ and that the reason we see ice formation at temperatures above approximately $-35^{\circ} \mathrm{C}$ is due to the presence of impurity particles. ${ }^{1}$ This is known as heterogeneous nucleation. It is also known that different impurity particles aid ice formation with different efficiencies, for example, feldspar mineral particles have been found to be better ice nucleating agents than clay mineral particles. ${ }^{2}$ What is severely lacking, however, is a comprehensive understanding of heterogeneous ice nucleation: we simply do not understand which properties of a material affect its ability to nucleate ice. Given the ubiquity of ice formation and its important role in the atmospheric, geological, and biological sciences, as well as the problems it can cause in the food, transport, and energy industries, acquiring a full understanding of heterogeneous ice nucleation remains a major challenge in urgent need of address. ${ }^{3}$

Experimentally, ice nucleation remains a challenge to study as it occurs on small time and length scales, and computer simulation therefore provides a useful tool when investigating both homogeneous ${ }^{4-9}$ and heterogeneous ice nucleation. ${ }^{10-15}$ Recent studies have used molecular dynamics simulation in combination with coarse grained models to probe the mechanisms of heterogeneous ice nucleation. In particular, Lupi et al. ${ }^{13,14}$ have investigated the effect of graphitic surfaces on ice nucleation and have found that the extent of layering of interfacial water correlates with the freezing temperature; in our first paper in the series, ${ }^{12}$ on the other hand, we focused on how the hydrophilicity of an hexagonal surface that acts as a

\footnotetext{
a)Electronic mail: angelos.michaelides@ucl.ac.uk
}

template for the basal face of ice affects the rate and found that an optimal interaction strength between the water and the surface exists. In this second article, we present further results from simulations of ice nucleation in the presence of "graphitic" surfaces. Unlike Ref. 14, where the primary aim was to understand ice nucleation on soot particles, here we are not attempting to model actual graphitic surfaces. Rather, in this study, we wish to exploit the smoothness of the potential experienced by the water molecules at such surfaces and compare to the results obtained in the first paper in this series, ${ }^{12}$ where the hexagonal surface under investigation presented distinct adsorption sites for the interfacial water molecules. We wish to emphasize that we are using simplified model surfaces in order to understand possible general trends that may underlie heterogeneous ice nucleation and we therefore probe a far greater range of hydrophilicities of these "graphitic" surfaces than previously considered by Lupi et al.

The aim of the first paper in this series was to demonstrate that, by understanding the molecular mechanism by which a surface facilitates ice formation, we could manipulate the surface to exert a degree of control over the rate. The primary purpose of this second article is to discuss the results of the first paper in the broader context of previous simulations on heterogeneous ice nucleation (in particular with respect to the recent work of Lupi et al. ${ }^{13,14}$ ). In what follows, we will find that the graphitic surfaces also exhibit an optimal interaction strength with water for promoting ice nucleation, which coincides with the optimal interaction strength found for the hexagonal surfaces presented in the first paper in this series. ${ }^{12} \mathrm{We}$ will also see that the previously suggested layering mechanism ${ }^{13,14}$ requires slight modification to be applied to strongly adsorbing surfaces and that the in-plane structure of the interfacial water molecules can affect the layering mechanism. This suggests that the layering mechanism cannot be used to explain the ice nucleating ability of surfaces in general. Finally, we discuss the 
origin of the observed optimal interaction strength and suggest a rule-of-thumb for relating the surface hydrophilicity to the ice nucleating efficiency.

\section{METHODS}

\section{A. Systems and force fields}

We have investigated heterogeneous ice nucleation in the presence of a rigid graphene nanoflake (GNF) of varying hydrophilicity, which is totally immersed in water as shown in Fig. 1. In this context, an increase in hydrophilicity is synonymous with an increase in the interaction strength between a water molecule and the surface. The interaction of water with the GNF was modeled using the two-body part of the Stillinger-Weber (SW) potential in the same manner as Lupi et al. ${ }^{13,14}$ The GNF consisted of 217 carbon atoms, with a carbon-carbon bond-length of $0.142 \mathrm{~nm}$ (perfect edges were assumed). The diameter of the GNF was approximately $2.5 \mathrm{~nm}$ to enable direct comparison to the results presented in the first paper in this series. As in Refs. 13 and 14, we used $\sigma_{\mathrm{SW}}$ $=0.32 \mathrm{~nm}$ to define the range of the water-carbon interaction (the functional form of the $\mathrm{SW} / \mathrm{mW}$ potential is given elsewhere $\left.{ }^{16}\right)$. The interaction strength was tuned by varying $\epsilon_{\mathrm{SW}}$. We note here that for the graphitic surfaces in Ref. 14, values in the range $0.12 \leq \epsilon_{\mathrm{SW}} \leq 0.2 \mathrm{kcal} / \mathrm{mol}$ were investigated; as this work is concerned with trying to obtain general understanding rather than modeling a specific system, we have broadened this range to $0.06 \leq \epsilon_{\mathrm{SW}} \leq 1.5 \mathrm{kcal} / \mathrm{mol}$. The total energy after geometry optimization of a single water molecule at the center of the GNF was used to define the water adsorption energy to the surface $E_{\text {ads }}$ (the water molecule optimized to a height $0.276 \mathrm{~nm}$ above the carbon atoms in the center of a graphene ring). No interaction was defined between the carbon atoms

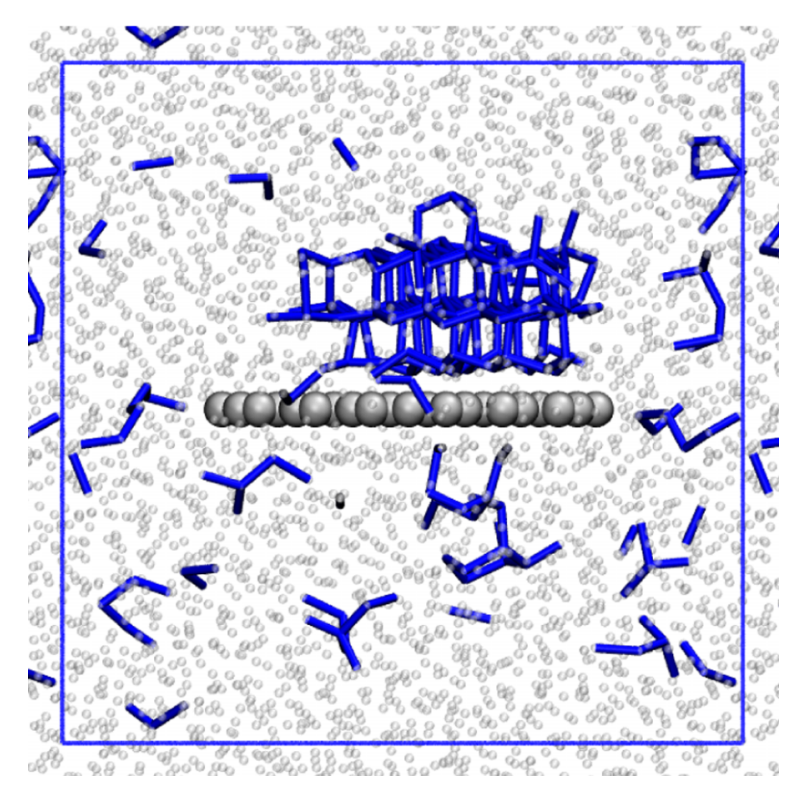

FIG. 1. A typical simulation of heterogeneous ice nucleation. The GNF is shown by large silver spheres and ice-like molecules are shown by blue lines. The remaining liquid-like water molecules are shown by small gray spheres. The box shows the boundary of the simulation cell and periodic boundary conditions are used throughout.
TABLE I. Dependence of the adsorption energy $E_{\text {ads }}$ on the water-carbon interaction strength $\epsilon_{\mathrm{SW}}$.

\begin{tabular}{cc}
\hline \hline$\epsilon_{\mathrm{SW}}(\mathrm{kcal} / \mathrm{mol})$ & $E_{\text {ads }}(\mathrm{kcal} / \mathrm{mol})$ \\
\hline 0.06 & 0.800 \\
0.13 & 1.734 \\
0.21 & 2.801 \\
0.29 & 2.868 \\
0.37 & 4.935 \\
0.45 & 6.002 \\
0.56 & 7.469 \\
0.67 & 8.936 \\
0.72 & 9.603 \\
0.80 & 10.669 \\
0.88 & 11.736 \\
1.00 & 13.337 \\
1.12 & 14.937 \\
1.31 & 17.471 \\
1.50 & 20.005 \\
\hline \hline
\end{tabular}

as their equations of motion were not integrated. In Ref. 14, it was discussed how one can also vary the hydrophilicity of such graphitic surfaces by introducing hydroxyl-like groups, which lead to different conclusions regarding how the hydrophilicity affects the rate of ice nucleation. The results presented in this article may help to understand this discrepancy, a point that we will return to in Sec. III B. To aid comparison with the work of Lupi et al., ${ }^{13,14}$ Table I shows how $E_{\text {ads }}$ depends upon $\epsilon_{\mathrm{SW}}$.

\section{B. Simulation settings}

All simulations were performed using the LAMMPS simulation package ${ }^{17}$ and the coarse grained $\mathrm{mW}$ model for water. ${ }^{16}$ The approximate diameter of a $\mathrm{mW}$ water molecule is $0.28 \mathrm{~nm}$, as estimated from the radial distribution function. ${ }^{16}$ The velocity Verlet algorithm was used to propagate the equations of motion of the water molecules, using a $10 \mathrm{fs}$ time step. In all simulations, $2944 \mathrm{~mW}$ molecules were used and periodic boundary conditions were applied in all three dimensions. As discussed in the first paper in this series, ${ }^{12}$ this system size is sufficiently large that finite size effects do not pose a serious problem at this temperature. This includes both increasing the number of water molecules and using a slab geometry (our results for the GNF are consistent with those obtained with a graphitic slab by Lupi et al. ${ }^{13,14}$ ). Temperature and pressure were maintained using the Nosé-Hoover thermostat and barostat (with a chain length of 10) with relaxation times of $1 \mathrm{ps}$ and $2 \mathrm{ps}$, respectively. A $100 \mathrm{~ns}$ trajectory was first performed at $290 \mathrm{~K}$ and $1 \mathrm{bar}$, from which initial configurations were drawn (different initial configurations were separated by at least $5 \mathrm{~ns}$ in the high temperature trajectory). At the start of the nucleation simulations, velocities for the water molecules were drawn randomly from a Maxwell-Boltzmann distribution to give an initial temperature of $205 \mathrm{~K}$. Simulations were stopped after $500 \mathrm{~ns}$ if nucleation did not occur. To detect "ice-like" molecules, we have used the CHILL algorithm of Moore et al. ${ }^{18}$ Rates were extracted in the same manner as in the first paper in the series ${ }^{12}$ and are directly comparable, since we have used the same simulation protocol. 


\section{Analysis}

For each value of $E_{\text {ads }}$, an extra simulation of $10 \mathrm{~ns}$ was performed at $215 \mathrm{~K}$ and 1 bar (following a 1 ns equilibration period from a $290 \mathrm{~K}$ configuration). Similarly, a set of $10 \mathrm{~ns}$ simulations were performed at $225 \mathrm{~K}$ and 1 bar for the face centered cubic nanoparticle investigated in the first paper in this series. ${ }^{12}$ (We refer to this nanoparticle as the "FCC-111 NP.") These higher temperature simulations were performed such that sufficient statistics in the liquid state could be obtained over the full range of $E_{\text {ads }}$ (i.e., to avoid crystallization over a $10 \mathrm{~ns}$ interval). We wish to emphasize that all simulations used to calculate the nucleation rate were obtained at $205 \mathrm{~K}$.

The layering of interfacial water was computed as

$$
L=\int_{0}^{z_{\text {bulk }}} \mathrm{d} z\left|\frac{\rho(z)}{\rho_{\text {bulk }}}-1\right|^{2},
$$

where $\rho(z)$ is the local water density at a height $z$ above the surface (see Fig. 2), $\rho_{\text {bulk }}$ is the density of bulk liquid water at $215 \mathrm{~K}$ (or $225 \mathrm{~K}$ ) and 1 bar (also obtained from a $10 \mathrm{~ns}$ simulation), and $z_{\text {bulk }}=1.8 \mathrm{~nm}$ is a height at which $\rho(z) \rightarrow \rho_{\text {bulk. }}$. We note that, where comparison could be made with the simulations at $205 \mathrm{~K}$, the value of $L$ appears to be rather insensitive to the temperature (differences are less than 1 unit) - the effect of changing $E_{\text {ads }}$ is by far the more dominant effect. The integration was performed using the Trapezium rule (Simpson's rule was also used, with a maximum discrepancy between the two methods of 3\% and agreement generally within $1 \%$ ). In computing $\rho(z)$, only water molecules in the column above the surface were considered (the radius of the column was $1.25 \mathrm{~nm}$ above both the GNF and the FCC-111 NP).

The probability density $P(x, y)$ of water molecules in the plane of the surface was computed for the water molecules in the contact and second layers above the surface. A water molecule was defined as being in the contact layer if $0 \leq z$ $<0.45 \mathrm{~nm}$ and in the second layer if $0.45 \leq z<0.8 \mathrm{~nm}$ as shown in Fig. 2(a). A similar analysis was also performed for the FCC-111 NP, with water molecules defined as being in the contact layer if $0 \leq z<0.35 \mathrm{~nm}$ and in the second layer if $0.35 \leq z<0.7 \mathrm{~nm}$, as shown in Fig. 2(b).

\section{RESULTS AND DISCUSSION}

\section{A. Nucleation rates and the role of interfacial layering}

In Fig. 3, we show how the nucleation rate $R$ varies with the hydrophilicity of the GNF. Specifically, we have plotted $\log _{10}\left(R / R_{\text {hom }}\right)$ against $E_{\text {ads }} / \Delta H_{\text {vap }}$, where $R_{\text {hom }}$ is the homogeneous nucleation rate and $\Delta H_{\text {vap }}$ is the heat of vaporization of bulk $\mathrm{mW}$ water $(10.65 \mathrm{kcal} / \mathrm{mol}$ at $298 \mathrm{~K}) .{ }^{16}$ We can clearly see that for the weakest interaction strength, the GNF has little effect on the nucleation rate. As $E_{\text {ads }}$ increases, the rate rapidly increases to reach a maximum at $E_{\text {ads }} / \Delta H_{\text {vap }} \approx 0.3-0.4$ that is approximately a factor 25 faster than homogeneous nucleation. We note here that a factor 25 increase in the rate would appear small when compared to experimental values, which often span many orders of magnitude. This is due to the fact
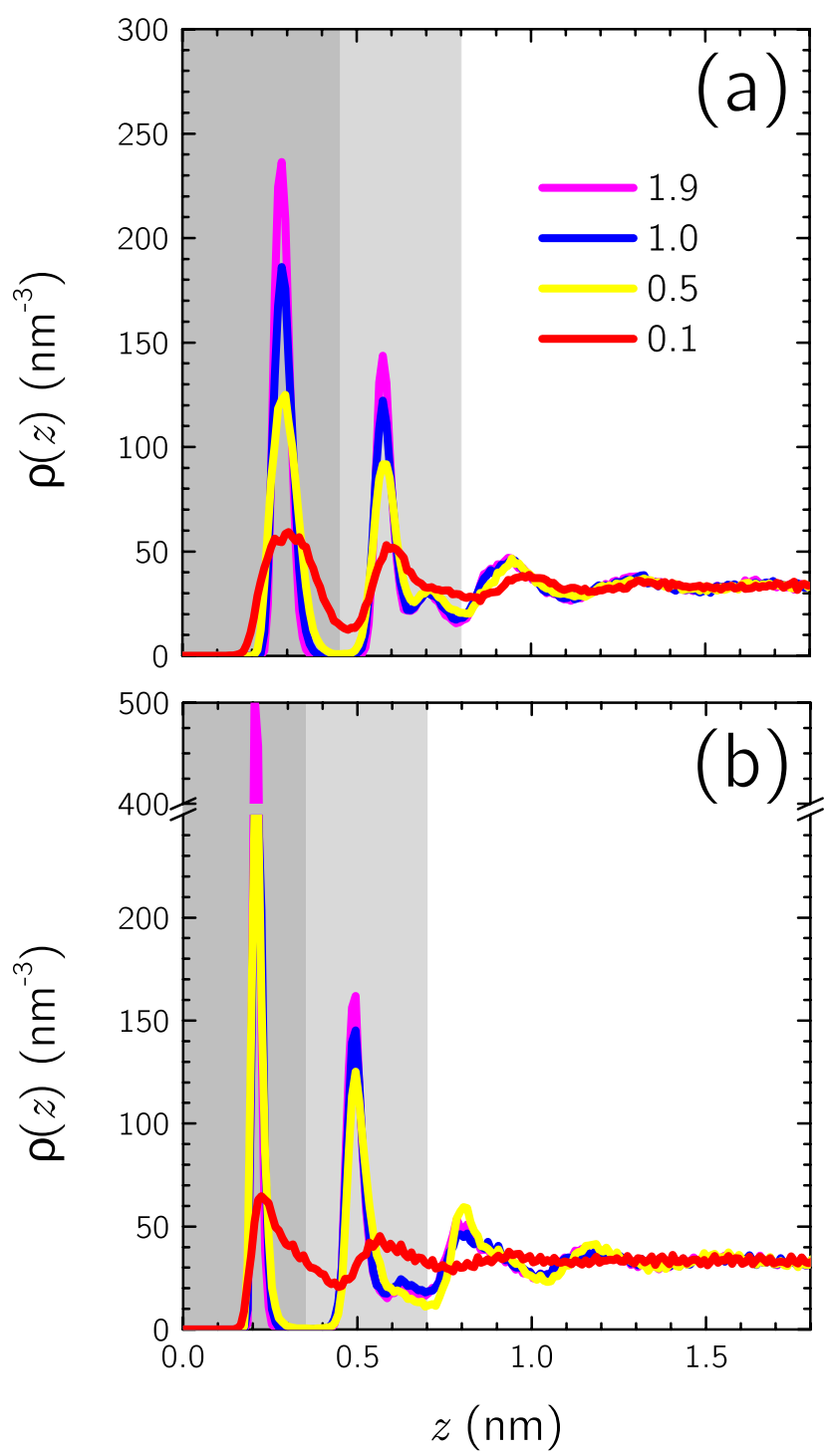

FIG. 2. Density profile $\rho(z)$ of water above the surface of the (a) GNF at $215 \mathrm{~K}$ and (b) the FCC-111 NP at $225 \mathrm{~K}$, for different values of $E_{\text {ads }} / \Delta H_{\text {vap }}$ (where $\Delta \boldsymbol{H}_{\text {vap }}$ is the heat of vaporization of liquid $\mathrm{mW}$ water at $298 \mathrm{~K}$ ). At both surfaces, water forms layers with the intensity and sharpness of the layers increasing with $E_{\text {ads }}$. The dark gray shaded region indicates water molecules defined as belonging to the first layer and the light gray shaded region water molecules defined as belonging to the second layer (see Sec. II C).

that we are operating at low temperatures so that we can directly compare to homogeneous nucleation. The effects of heterogeneous ice nucleation will become more pronounced at higher temperatures. Upon increasing $E_{\text {ads }}$ further, the rate steadily drops until $E_{\text {ads }} / \Delta H_{\text {vap }} \approx 1.0$ when the rate begins to steadily increase again. For the most strongly interacting GNF investigated, the rate is a little over 10 times that of homogeneous nucleation. We also show the results from Ref. 12 of the nucleation rate in the presence of the FCC-111 NP. Both the GNF and the FCC-111 NP exhibit a maximum in rate at $E_{\text {ads }} / \Delta H_{\text {vap }} \approx 0.3-0.4$, but differ in that at $E_{\text {ads }} / \Delta H_{\text {vap }} \approx 1.0$, the FCC-111 NP crosses from promoting to inhibiting rather than exhibiting a local minimum like the GNF. This crossover from promoting to inhibiting can be explained by an excess of favorable adsorption sites at the FCC-111 NP (shown in Fig. 4 and discussed in detail in Ref. 12). 


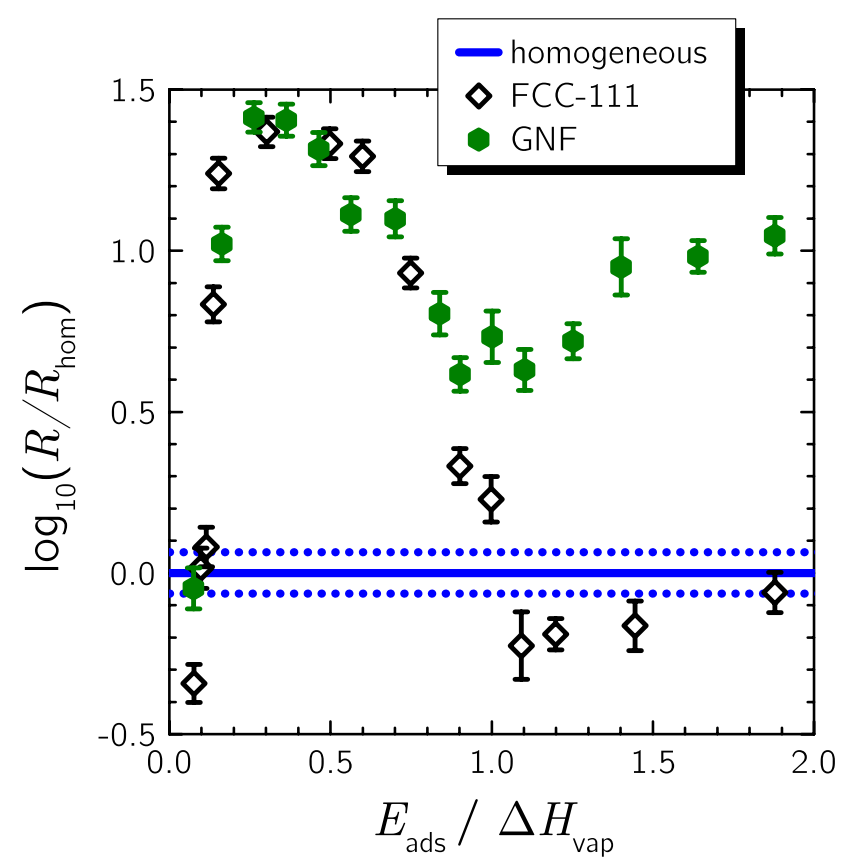

FIG. 3. Dependence of the heterogeneous nucleation rate on surface hydrophilicity. As $E_{\text {ads }}$ increases so too does the hydrophilicity. The homogeneous and FCC-111 data are taken from Ref. 12. Like the FCC-111 NP, the GNF also exhibits a maximum rate at $E_{\text {ads }} / \Delta H_{\text {vap }} \approx 0.3-0.4$, but, in contrast, exhibits a local minimum in the rate at $E_{\text {ads }} / \Delta H_{\text {vap }} \approx 1.0$.

To explain the ice nucleating ability of the graphitic surfaces such as those considered here, Lupi et al. found that the layering of interfacial water $L$ (see Eq. (1)) correlates with the ice nucleating ability. ${ }^{14}$ In Fig. 5(a), we show the dependence of $L$ on $E_{\text {ads }}$ for the GNF. Unsurprisingly, $L$ increases monotonically with $E_{\text {ads }}$ and we therefore cannot explain the observed non-monotonic dependence of $R$ on $E_{\text {ads }}$ seen in Fig. 3 simply by the extent of layering. Figs. 6(a) and 6(b) provide some insight into why this is the case, where we show typical structures of water in contact with the GNF at $215 \mathrm{~K}$ for $E_{\text {ads }} / \Delta H_{\text {vap }} \approx 0.25$ and $E_{\text {ads }} / \Delta H_{\text {vap }} \approx 1.9$, respectively. For values of $E_{\text {ads }}$ that yield the highest rates, such as in Fig. 6(a), water forms structures in the contact layer that resemble
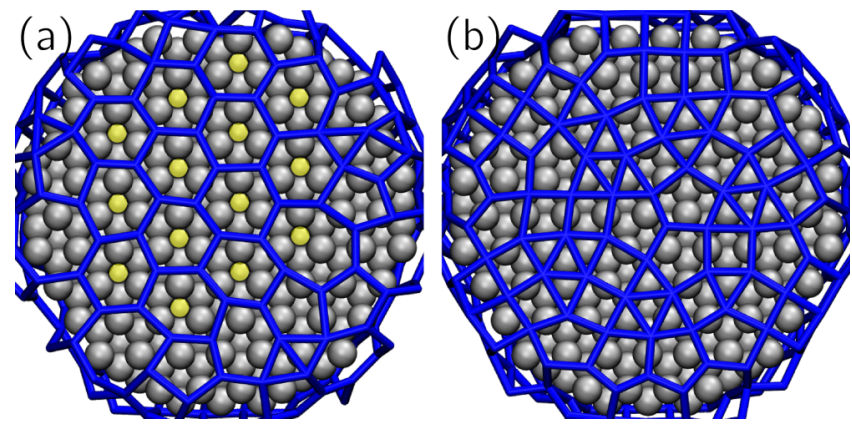

FIG. 4. Typical structures that form in the contact layer at the FCC-111 NP at $225 \mathrm{~K}$. The FCC-111 NP is shown in silver and the water molecules in blue. (a) FCC-111 NP with $E_{\text {ads }} / \Delta H_{\text {vap }} \approx 0.3$. A hexagonal overlayer, which is commensurate with the surface, that resembles ice is observed and facilitates ice formation at $205 \mathrm{~K}$. Unoccupied, or "excess," adsorption sites (highlighted by yellow circles) are present when this structure forms. (b) FCC-111 NP with $E_{\text {ads }} / \Delta H_{\text {vap }} \approx 1.9$. For this stronger interaction with the surface, the excess sites are occupied and the hexagonal structure resembling ice is no longer observed.
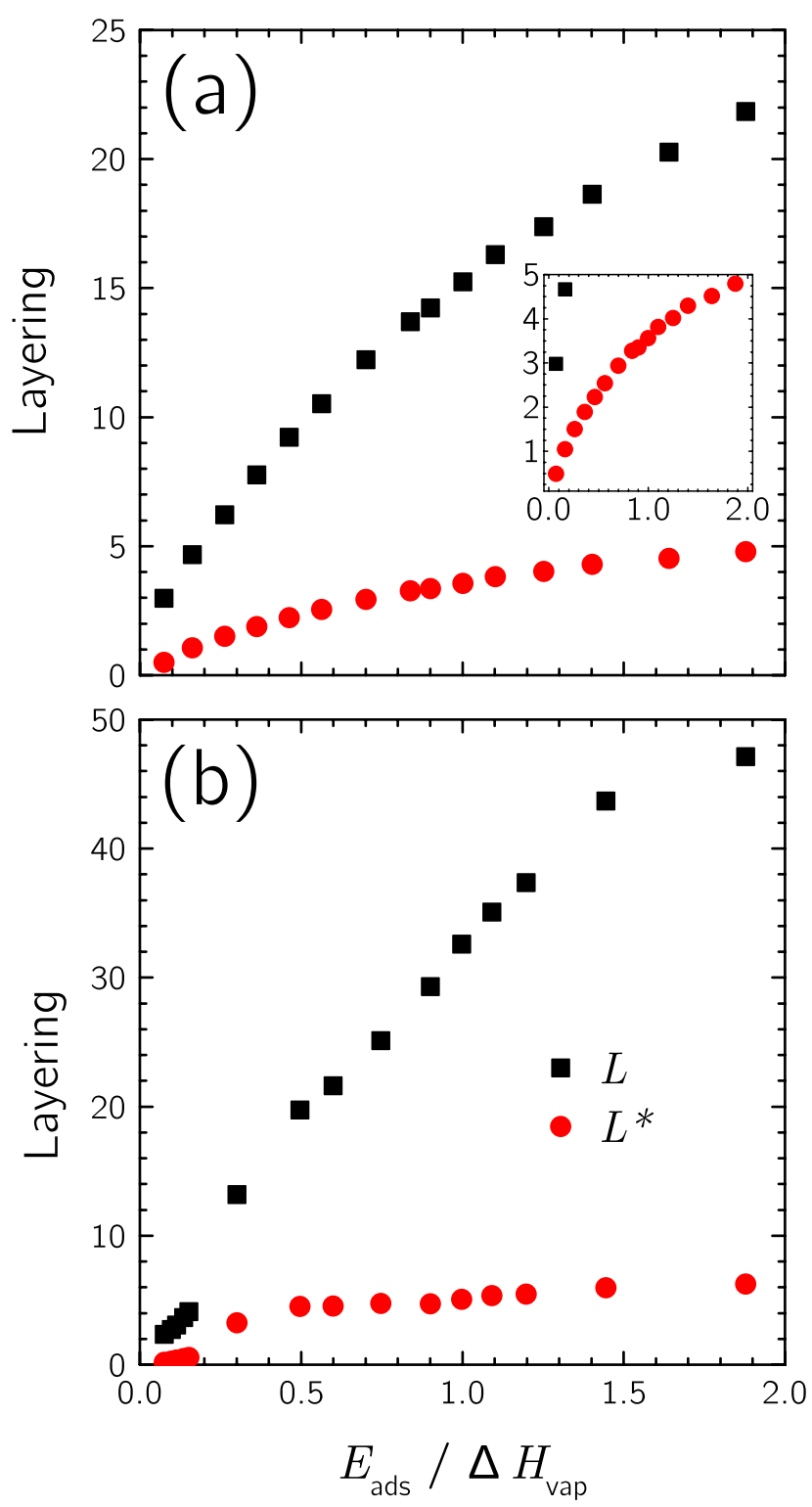

FIG. 5. Dependence of the extent of layering of interfacial water on $E_{\text {ads }}$. The black squares show the layering over the whole density profile $L$ as defined by Eq. (1), whereas the red circles show the layering excluding the contact layer $L^{*}$ as defined by Eq. (2). (a) Result for the GNF at $215 \mathrm{~K}$. Both $L$ and $L^{*}$ increase monotonically with $E_{\text {ads }}$, but $L$ does so much more rapidly. The inset contains the same data but with a smaller scale for the $y$-axis, which shows more clearly that $L^{*} \gtrsim 3$ only once $E_{\text {ads }} / \Delta H_{\text {vap }} \gtrsim 0.7$ (when $E_{\text {ads }} / \Delta H_{\text {vap }} \approx 0.1, L \approx 3$ and nucleation is not enhanced). (b) Results from the FCC-111 NP at $225 \mathrm{~K}$. Although $L$ is much greater at the FCC-111 NP than at the GNF, the values of $L^{*}$ are comparable.

the hexagonal structure of ice. Indeed, when ice formation is observed at $205 \mathrm{~K}$, it appears to be driven by the formation of such hexagonal patches in the contact layer, consistent with previous studies. ${ }^{13,14}$ In the case of the strongly adsorbing GNF shown in Fig. 6(b), it is clear that the number of water molecules in contact with the GNF has increased and that, rather than a hexagonal structure similar to ice, a structure consisting predominantly of smaller membered rings is now observed. This structure bears a strong resemblance to those seen in confined water layers under high pressures ${ }^{19,20}$ and can be understood as water maximizing its interaction to the surface with only a slight cost in hydrogen bond energy. ${ }^{10}$ 


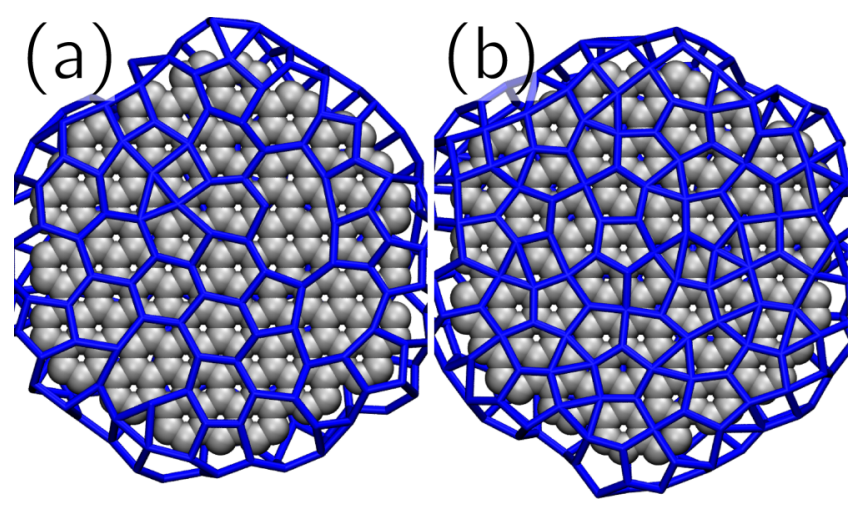

FIG. 6. Typical structures of water in the contact layer at the GNF at $215 \mathrm{~K}$. The GNF is shown in silver and the water molecules in blue. (a) GNF with $E_{\text {ads }} / \Delta H_{\text {vap }} \approx 0.25$. Patches of ice-like hexagons readily form in the contact layer and facilitate ice formation at $205 \mathrm{~K}$. (b) GNF with $E_{\text {ads }} / \Delta H_{\text {vap }} \approx 1.9$. At this more strongly adsorbing surface, the structure in the contact layer consists predominantly of smaller membered rings. This persists even after ice nucleation at $205 \mathrm{~K}$, which at this interaction strength occurs in the water layers above.

When ice formation is observed at this surface, it does so in the layers of water above the contact layer, with the structure in the contact layer remaining unchanged. Thus, for high values of $E_{\text {ads }}$, the contact layer is "inactive" with respect to nucleation.

The observation that the contact layer becomes inactive to ice nucleation for strong adsorption energies is enough to understand why we begin to see a decrease in the nucleation rate beyond $E_{\text {ads }} / \Delta H_{\text {vap }} \approx 0.3-0.4$. To explain the increase in rate beyond $E_{\text {ads }} / \Delta H_{\mathrm{vap}} \approx 1.0$, however, requires further analysis. To this end, we have computed the layering of interfacial water with contributions from the first layer excluded

$$
L^{*}=\int_{z_{0}}^{z_{\text {bulk }}} \mathrm{d} z\left|\frac{\rho(z)}{\rho_{\text {bulk }}}-1\right|^{2},
$$

where at the GNF, $z_{0}=0.45 \mathrm{~nm}$. In Fig. 5(a), we can see that $L^{*}$, like $L$, also increases monotonically with $E_{\text {ads }}$, but much more slowly. We can also see that the value of $L^{*}$ at $E_{\text {ads }} / \Delta H_{\text {vap }} \approx 1.9$ is similar to the value of $L$ at $E_{\text {ads }} / \Delta H_{\text {vap }} \approx 0.2$ and from Fig. 3, that these two adsorption energies yield similar rates (both approximately a factor 10 faster than homogeneous nucleation). It therefore seems that beyond $E_{\text {ads }} / \Delta H_{\text {vap }} \approx 1.0$, the extent of layering in the second layer of water and above becomes sufficient to promote ice nucleation. This can be seen more clearly in the inset of Fig. $5(\mathrm{a})$; bearing in mind that the most weakly interacting GNF yields $L \approx 3$ and does not promote ice nucleation, we can see that $L^{*}$ only begins to exceed this value for $E_{\text {ads }} / \Delta H_{\text {vap }}>0.7$.

\section{B. The layering mechanism depends upon the in-plane structure of water}

Section III A provides strong evidence in support of the layering mechanism, albeit with a slight modification to what was originally proposed. ${ }^{13,14}$ Conceptually, the layering mechanism is appealing and can perhaps be understood in terms of reducing the entropic barrier to nucleation: if water molecules are restricted to motion in a particular plane (with a density acceptable for ice nucleation), then the space that they can explore is effectively reduced by one dimension relative to the bulk liquid, which subsequently reduces the number of possible configurations that the water molecules can explore. This argument, however, implicitly assumes that the effective potential experienced by the water molecules within a layer is uniform.

For the graphitic surfaces investigated in this study, the assumption of a uniform effective potential within the layers is likely to be reasonable. Now consider the FCC-111 NP with $E_{\text {ads }} / \Delta H_{\text {vap }} \approx 1.9$ which, as can be seen in Fig. 3, does not promote ice nucleation. The GNF with similar $E_{\text {ads }}$, on the other hand, enhances ice nucleation by a factor $\sim 10$ relative to homogeneous nucleation. Furthermore, the layering (excluding contributions from the contact layer) above both of these surfaces is similar, with $L^{*} \approx 6$ for the FCC-111 NP and $L^{*} \approx 5$ for the GNF, as shown in Fig. 5(b) (a value of $z_{0}=0.35 \mathrm{~nm}$ is used in Eq. (2) for the FCC-111 NP). The difference in rates can be understood in terms of in-plane structure, as seen in Fig. 7, where we show $-\ln [P(x, y)]$, where $P(x, y)$ is the probability density of water molecules in the plane of the surface at $215 \mathrm{~K}$, both for the contact layer and the second layer above the surface (see Sec. II C). At the FCC$111 \mathrm{NP}$, the water molecules bind at distinct adsorption sites (see Fig. 7(b)) and do not diffuse over the $10 \mathrm{~ns}$ time scale of the simulation. Importantly, this impacts upon the structure of the water molecules in the second layer (see Fig. 7(d)), which tend to be found directly above those in the contact layer. In contrast, the water molecules in contact with the GNF (see Fig. 7(a)) do not adsorb at particular adsorption sites and are not immobile like at the FCC-111 NP, resulting in a smearing-out of $P(x, y)$. Accordingly, $P(x, y)$ for the second layer above the GNF (see Fig. 7(c)) is much smoother than at the FCC-111 NP. The smoothness of $P(x, y)$ for the second layer above the GNF means that the water molecules can rearrange to form ice-like structures, whereas the corrugation in $P(x, y)$ for the second layer above the FCC-111 NP appears to frustrate the water molecules, hindering ice formation.

In Refs. 13 and 14, the general applicability of the layering mechanism was left as an open question. By vastly broadening the range of hydrophilicities investigated and monitoring the response of the ice nucleation rate in the presence of two model surfaces, we are able to elucidate when the layering mechanism may be important. The results of our simulations show that the extent of layering can be important for ice nucleation, but that if the coverage of water at the surface is too high, then the contributions of the contact layer to the layering should be omitted. Furthermore, the importance of layering is dependent upon the water molecules experiencing a rather uniform effective potential within the layers. The extent of layering is therefore not a universal descriptor for the ice nucleating ability of surfaces.

We finish this section with a comment regarding the manner by which the hydrophilicity of the GNF has been tuned. In this study, this has been achieved by uniformly changing the value of $\epsilon_{\mathrm{SW}}$. In Ref. 14, however, Lupi and Molinero also investigated the effect of introducing hydroxyl-like groups (with higher concentrations of hydroxyl-like groups corresponding to more hydrophilic surfaces). They found that increasing the hydrophilicity in such a manner was in fact 

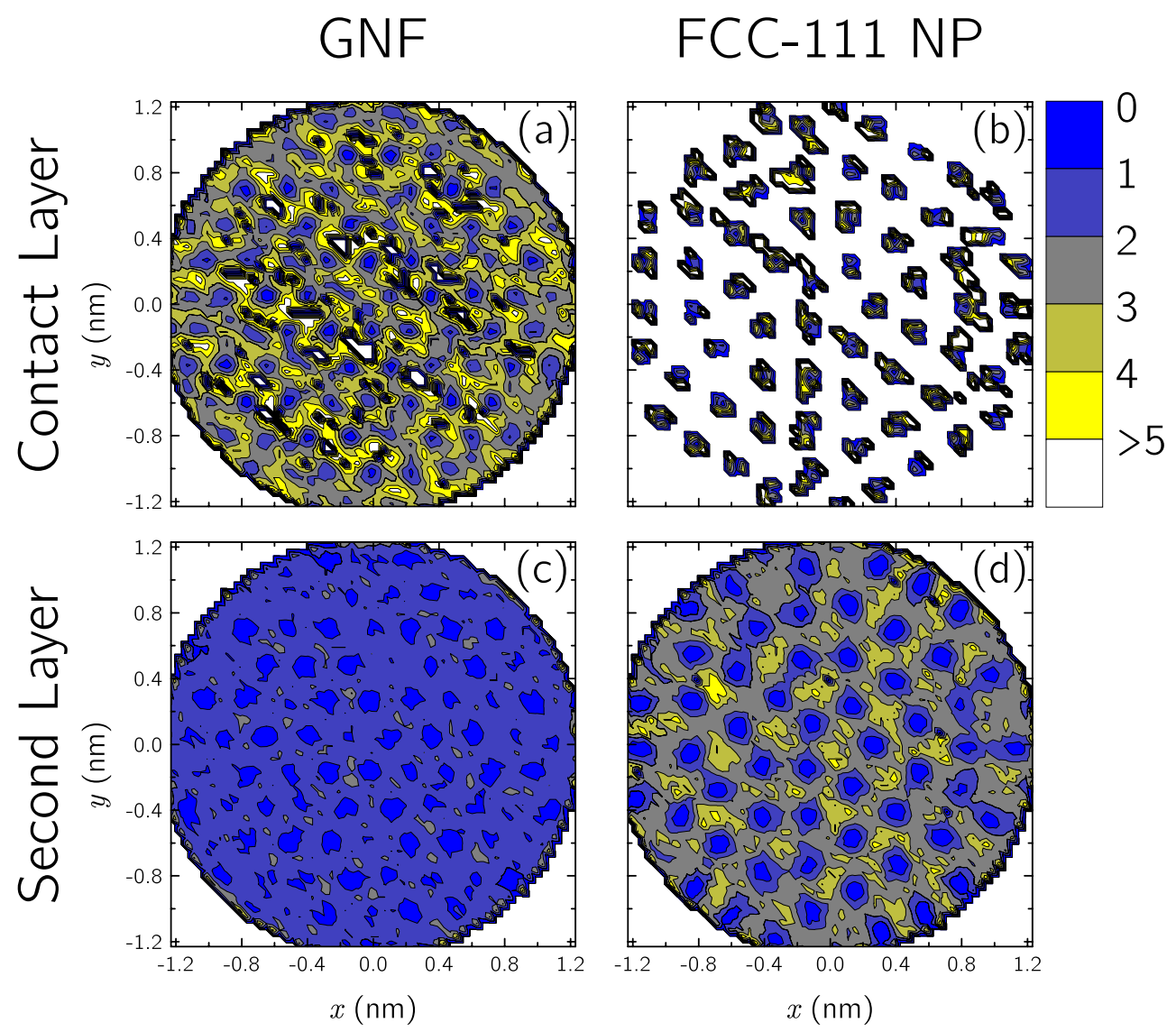

FIG. 7. In-plane distribution of water molecules above the surface, plotted as $-\ln [P(x, y)]$ (see Sec. II C) with $E_{\text {ads }} / \Delta H_{\text {vap }} \approx 1.9$ at $215 \mathrm{~K}$. (a) and (b) show the contact layer at the GNF and FCC-111 NP, respectively. (c) and (d) show the second layer above the GNF and FCC-111 NP, respectively. Unlike at the GNF, the water molecules in the contact layer with the FCC-111 NP are bound at specific adsorption sites and do not diffuse over the time scale of the simulation (10 ns). The water molecules in the second layer above the FCC-111 NP consequently exhibit greater structure than those in the second layer above the GNF.

detrimental to the rate, while changing $\epsilon_{\mathrm{SW}}$ over the range $0.12-0.2 \mathrm{kcal} / \mathrm{mol}$ enhanced ice nucleation, consistent with our findings (see Table I). The results presented in this section may reconcile the discrepancy between the two approaches: by uniformly increasing $\epsilon_{\mathrm{SW}}$ over this relatively narrow range, ice nucleation is enhanced due to an increase in $L$ as the water molecules are still moving in a relatively smooth effective potential; the introduction of hydroxyl-like groups, on the other hand, is likely to localize water molecules at certain positions at the surface, which may cause a similar frustration to that described at the FCC-111 NP, if the spatial arrangement of hydoxyl-like groups is not conducive to ice nucleation.

\section{The effect of surface hydrophilicity on heterogeneous ice nucleation}

In this section, we will discuss the observation that the GNF and the FCC-111 NP both have optimal interaction strengths at $E_{\text {ads }} / \Delta H_{\text {vap }} \approx 0.3-0.4$ in more detail. If the interaction between the water and the surface is too weak, the induced structural differences from the bulk liquid are not significant enough to promote ice nucleation at either the GNF or the FCC-111 NP. What is more intriguing is why the ice nucleation rate at both surfaces decreases beyond $E_{\text {ads }} / \Delta H_{\text {vap }} \approx 0.3-0.4$. Despite the differences in surface topography (the GNF can be considered a smooth surface, whereas the FCC-111 NP presents distinct adsorption sites), both surfaces share a common feature: they can both accommodate water coverages that are higher than that when ice forms at the surface. This has been demonstrated qualitatively in Figs. 4 and 6. Fig. 8 provides quantitative evidence for this statement, where we show the lateral density of water molecules $\sigma$ in the contact and second layers,

$$
\sigma=\int_{\text {layer }} \mathrm{d} z \rho(z)
$$

where the integral runs over the layer of interest (see Sec. II C). Note that $\sigma$ can also be computed explicitly by counting the average number of water molecules in a given layer: such an approach also gives information regarding the fluctuations of $\sigma$. For the GNF, shown in Fig. 8(a), we can see that $\sigma$ for the contact layer steadily increases with $E_{\text {ads }}$, whereas for the second layer, although increasing slightly initially, $\sigma$ is essentially constant. In terms of layering, this means $L^{*}$ is increasing primarily through a narrowing of the second peak in $\rho(z)$, whereas $L$ also has a contribution from an increased number of water molecules at the surface. To a lesser extent, the same is true for the FCC-111 NP, shown in Fig. 8(b), although some variation in $\sigma$ for the second layer is also observed.

We have also marked in Figs. 8(a) and 8(b) the water coverage of ice that forms at the surface for the GNF and the FCC-111 NP, respectively. ${ }^{21}$ We can clearly see that for $E_{\text {ads }} / \Delta H_{\text {vap }} \approx 0.3-0.4$, the value of $\sigma$ in the contact layer is comparable to that of ice that forms at the surface. Thus, as 

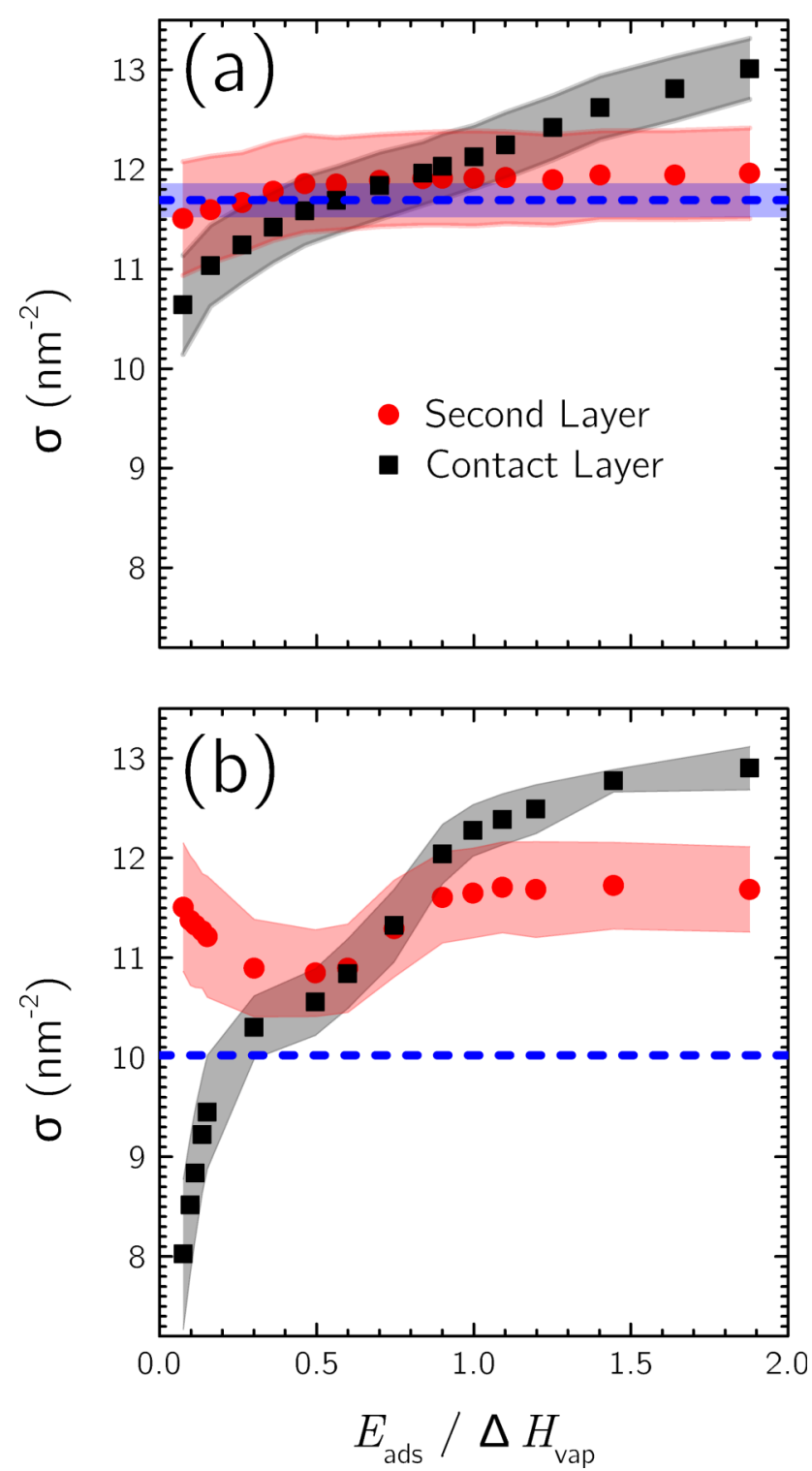

FIG. 8. Dependence of the lateral density of water molecules $\sigma$ on $E_{\text {ads }}$ for (a) the GNF at $215 \mathrm{~K}$ and (b) the FCC-111 NP at $225 \mathrm{~K}$. The blue dashed lines indicate the water coverage of ice that forms at both surfaces (the blue shaded area in (a) indicates the standard deviation of a sample average taken over the range $0.16 \leq E_{\text {ads }} / \Delta H_{\text {vap }} \leq 0.56$ ). The black and red shaded areas indicate the standard deviation in the measured values of $\sigma$ for the contact and second layers, respectively. When $E_{\text {ads }} / \Delta H_{\text {vap }} \approx 0.3-0.4$, the coverage in the contact layer is close to the water coverage of ice that forms.

$E_{\text {ads }}$ increases further, it becomes increasingly favorable for water to adsorb to the surface to the detriment of ice nucleation occurring in the contact layer. We therefore suggest a rule-ofthumb for the role of surface hydrophilicity in ice nucleation: for surfaces that can accommodate water coverages higher than that required by ice, binding to the surface should not be too strong, with optimal adsorption energies approximately $30 \%-40 \%$ the heat of vaporization of liquid water. We must stress that the importance of surface hydrophilicity will depend upon the water coverage that the surface can accommodate. As we have shown in the first paper in this series, ${ }^{12}$ surfaces with $E_{\text {ads }} / \Delta H_{\text {vap }} \gg 0.4$ can exhibit excellent ice nucleating efficiency, provided that the coverage of water at the surface does not exceed that of ice. For example, surfaces that resemble the surface of ice itself may also favor more open structures, such that this rule-of-thumb cannot be applied. ${ }^{22}$

\section{CONCLUSIONS}

We have presented computer simulations of heterogeneous ice nucleation in the presence of a graphene nanoflake immersed in water and investigated how its hydrophilicity affects the nucleation rate. The results of our simulations in part support the previously proposed layering mechanism of Lupi et al.,$^{13,14}$ although we have seen that for strongly adsorbing surfaces, the increased layering, due to a higher coverage of water molecules, is detrimental to ice nucleation. Under such conditions, by excluding the contribution of the water molecules in contact with the surface, the extent of layering is, however, still found to correlate with the heterogeneous nucleation rate. It has also been demonstrated that the layering mechanism is not universal, as surfaces that exhibit similar degrees of interfacial layering can yield vastly different rates. We attribute this finding to the extent that the surface affects the in-plane structure of the water molecules: for surfaces where the water molecules move in a smooth effective potential, like the graphitic surfaces investigated in this article, the extent of layering describes the nucleation rate well; for surfaces that present distinct adsorption sites, such as the FCC (111) surface investigated in Ref. 12, the induced structure can frustrate ice nucleation not only in the contact layer but also in the layer above.

We have observed that an optimal interaction strength between the graphene nanoflake and water for ice nucleation exists, and that this coincides with the optimal interaction strength found for the FCC (111) surface investigated previously. ${ }^{12}$ This behavior has been rationalized by noting that both surfaces are able to accommodate coverages of water that are higher than that when ice forms at these surfaces. We have proposed a rule-of-thumb, which states that in order to nucleate ice efficiently, a surface should not bind water too strongly if it can accommodate high coverages of water. Such insight may prove useful when trying to predict a material's ice nucleating ability, especially as the coverage of liquid water should be obtainable through, e.g., surface X-ray diffraction experiments.

\section{ACKNOWLEDGMENTS}

We are grateful to the London Centre for Nanotechnology and UCL Research Computing for computation resources, and the UK's national high performance computing service (from which access was obtained via the UK's Material Chemistry Consortium, EP/F067496). S.M.K. was supported fully by the U.S. Department of Energy, Office of Basic Energy Sciences, Division of Chemical Sciences, Geosciences \& Biosciences. Pacific Northwest National Laboratory (PNNL) is a multiprogram national laboratory operated for DOE by Battelle. S.J.C. was supported by a student fellowship funded jointly by UCL and BES. A.M. is supported by the European Research Council under the European Union's Seventh Framework Programme (FP/2007-2013)/ERC Grant Agreement No. 616121 (HeteroIce project) and the Royal Society through a Royal Society Wolfson Research Merit Award. 
${ }^{1}$ B. J. Murray, D. O’Sullivan, J. D. Atkinson, and M. E. Webb, Chem. Soc. Rev. 41, 6519 (2012).

${ }^{2}$ J. D. Atkinson, B. J. Murray, M. T. Woodhouse, T. F. Whale, K. J. Baustian, K. S. Carslaw, S. Dobbie, D. O'Sullivan, and T. L. Malkin, Nature 498, 355 (2013).

${ }^{3}$ T. Bartels-Rausch, Nature 494, 27 (2013).

${ }^{4}$ E. B. Moore and V. Molinero, Nature 479, 506 (2011).

${ }^{5}$ A. Reinhardt and J. P. K. Doye, J. Chem. Phys. 136, 054501 (2012).

${ }^{6}$ E. B. Moore and V. Molinero, J. Chem. Phys. 132, 244504 (2010).

${ }^{7}$ T. Li, D. Donadio, G. Russo, and G. Galli, Phys. Chem. Chem. Phys. 13, 19807 (2011)

${ }^{8}$ T. Li, D. Donadio, and G. Galli, Nat. Commun. 4, 1887 (2013).

${ }^{9}$ E. Sanz, C. Vega, J. R. Espinosa, R. Caballero-Bernal, J. L. F. Abascal, and C. Valeriani, J. Am. Chem. Soc. 135, 15008 (2013).

${ }^{10}$ S. J. Cox, S. M. Kathmann, J. A. Purton, M. J. Gillan, and A. Michaelides, Phys. Chem. Chem. Phys. 14, 7944 (2012).

${ }^{11}$ S. J. Cox, Z. Raza, S. M. Kathmann, B. Slater, and A. Michaelides, Faraday Discuss. 167, 389 (2013)

${ }^{12}$ S. J. Cox, S. M. Kathmann, B. Slater, and A. Michaelides, J. Chem. Phys. 142, 184704 (2015).
${ }^{13}$ L. Lupi, A. Hudait, and V. Molinero, J. Am. Chem. Soc. 136, 3156 (2014).

${ }^{14}$ L. Lupi and V. Molinero, J. Phys. Chem. A 118, 7330 (2014).

${ }^{15}$ S. A. Zielke, A. K. Bertram, and G. N. Patey, "A molecular mechanism of ice nucleation on model AgI surfaces," J. Phys. Chem. B (published online).

${ }^{16}$ V. Molinero and E. B. Moore, J. Phys. Chem. B 113, 4008 (2009).

${ }^{17}$ S. Plimpton, J. Comput. Phys. 117, 1 (1995).

${ }^{18}$ E. B. Moore, E. de la Llave, K. Welke, D. A. Scherlis, and V. Molinero, Phys. Chem. Chem. Phys. 12, 4124 (2010).

${ }^{19}$ J. C. Johnston, N. Kastelowitz, and V. Molinero, J. Chem. Phys. 133, 154516 (2010).

${ }^{20}$ Q. Lu, J. Kim, J. D. Farrell, D. J. Wales, and J. E. Straub, J. Chem. Phys. 141, 18C525 (2014).

${ }^{21}$ Due to the different surface topographies, the coverage when ice forms is different for the GNF and the FCC-111 NP. In the case of the latter, $\sigma$ was calculated assuming 2/3-monolayer coverage of available adsorption sites at the (111) surface (the lattice constant of the FCC lattice was $0.392 \mathrm{~nm}$ ). For the GNF, the average projection of the second nearest neighbor distance onto the plane of the surface was measured, which was then used to compute $\sigma$.

${ }^{22}$ A. Reinhardt and J. P. K. Doye, J. Chem. Phys. 141, 084501 (2014). 